\title{
LOW POWER FSK DEMODULATOR
}

\author{
Saurav Singh ${ }^{1}$ \\ ${ }^{I}$ Student, Electronics and Communications, Maharaja Surajmal Institute of Technology, New Delhi, India
}

\begin{abstract}
Frequency shift keying (FSK) is the most common form of digital modulation in the high-frequency radio spectrum. The demodulation of Binary FSK involves complex operations on the modulated signal like reconstruction of carrier signal and multiplication of carrier signal with the modulated signal. In this paper, BFSK is realized as two separate ASKs and a much simpler incoherent method is proposed for demodulation of BFSK modulated signal. Since power consumption is one of the main factors in electronic devices, low power devices are always demanding. Power Consumption could be minimized by reducing the actual block diagram of the system by opting an alternative approach to achieve the same output signal. The same BFSK demodulator can be used to demodulate an ASK modulated signal if it follow certain criteria's.
\end{abstract}

Key Words: FSK, ASK, Demodulator, filters, MATLAB

\section{INTRODUCTION}

Binary FSK is a modulation scheme typically used to send digital information between digital equipment such as teleprinters and computers. The data are transmitted by shifting the frequency of a continuous carrier in a binary manner to one of the two discrete frequencies. One frequency level is called as 'Mark' and the other is called as 'Space'. The mark and space represents binary one and zero respectively. [1]

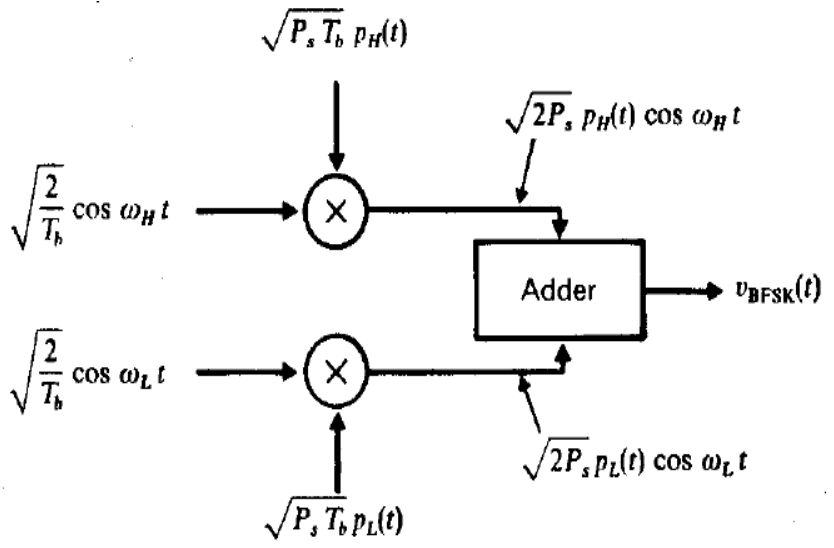

Fig -1: BFSK Modulation [2]

The BFSK can be visualized as sum of two ASK modulated waves. In Amplitude Shift Keying modulation technique, the carrier frequency is switched $\mathrm{ON}$ and OFF by the modulating binary data. When a one is to be transmitted, the carrier signal is switched $\mathrm{ON}$ and when a zero is to be transmitted, the carrier signal is switched OFF. BFSK signal can be generated using ASK modulation technique. If we modulate (ASK) the data with higher frequency carrier signal and inverted data with lower frequency carrier signal and add the two modulated signals, we will get FSK modulated signal. Figure 2 illustrates the above explanation.
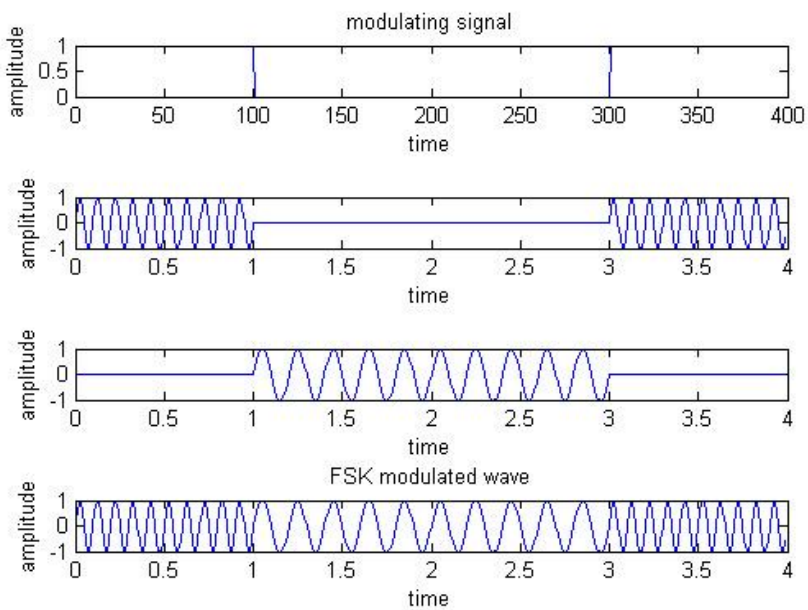

Fig -2: FSK from two ASK waveforms

To demodulate the BFSK signal by conventional methods, complex operations like multiplication of higher and lower frequency carrier signals are required. We also need to reconstruct the carrier signals for coherency.

In this paper a B-FSK demodulator is proposed. In ASK modulation, the carrier frequency is transmitted when the data is HIGH and no carrier frequency is transmitted when the data is LOW. On the other hand, in FSK high frequency carrier $\left(\mathrm{S}_{\mathrm{H}}(\mathrm{t})\right)$ is transmitted when data is $\mathrm{HIGH}$ and low frequency carrier $\left(\mathrm{S}_{\mathrm{L}}(\mathrm{t})\right)$ is transmitted when data is LOW.

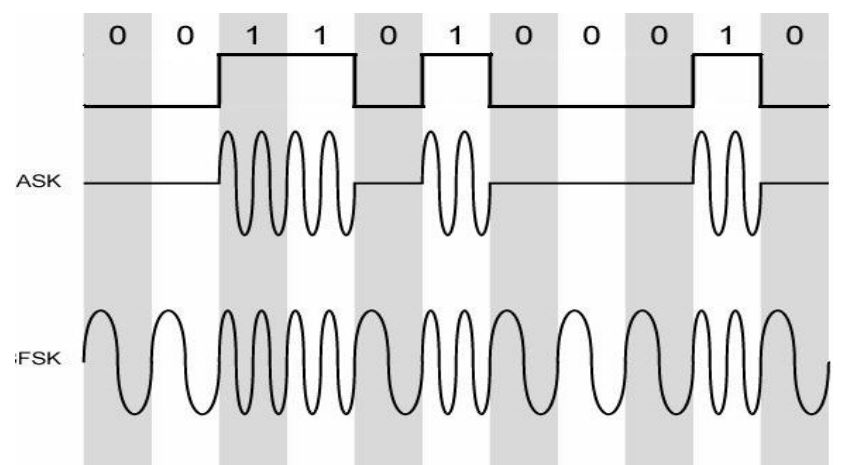

Fig -3: Comparision between ASK and FSK 


\subsection{Conventional BFSK Demodulation Technique}

The FSK can be viewed as two interleaved ASK signals with carrier frequencies $\mathrm{w}_{\mathrm{H}}$ and $\mathrm{w}_{\mathrm{L}}$, respectively. Therefore, FSK can be detected coherently or non-coherently. In noncoherent detection, the incoming signal is applied to a bank of two filters tuned to $\mathrm{w}_{\mathrm{H}}$ and $\mathrm{w}_{\mathrm{L}}$. Each filter is followed by envelop detector (Fig 4). The outputs of the two envelop detectors are sampled and compared. FSK can be detected coherently by generating two references of frequencies $w_{H}$ and $\mathrm{w}_{\mathrm{L}}$, and demodulating the received signal by two demodulators using the two carriers and then comparing the outputs of the two demodulators as shown in Fig 4. [3]
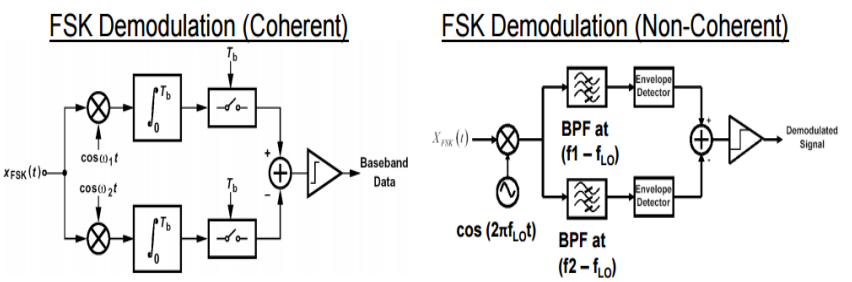

Fig -4: FSK Demodulation, Coherent and Non-Coherent Methods

\section{PROPOSED DEMODULATION METHOD}

If we use a BPF such that lower frequency carrier signal $\left(\mathrm{S}_{\mathrm{L}}(\mathrm{t})\right)$ lies in stopband and higher frequency carrier signal $\left(\mathrm{S}_{\mathrm{H}}(\mathrm{t})\right)$ lies in passband, then FSK signal can be effectively converted into ASK signal. An ASK demodulator can be used to recover the data. This way, we do not have to use the carrier recovery circuit at the receiver end (if we compare with the coherent demodulation method). Overall number of blocks are also reduced as the signal processing is done in a single stream rather than parallel processing for lower and higher carrier components of the BFSK signal. Further, if the carrier frequency of a ASK modulated signal lies in the passband of the Bandpass Fillter, the same circuit can be used to demodulate an ASK modulated signal also. The BPF can also be used to remove RF noise. The order of the BPF depends on the frequency separation between the two carrier signals in FSK.

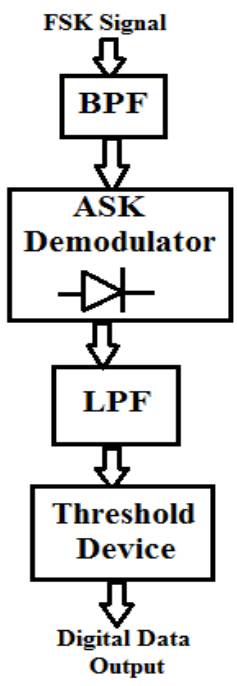

Fig -5: Proposed Block Diagram

\subsection{Advantages}

- Circuit is simpler to implement.

- Less number of blocks required, thus more power efficient system.

- No complex operations like multiplication or carrier recovery involved.

- It can also demodulate ASK modulated signals.

\subsection{Disadvantages}

- There is a phase lag in the output due to delayed response of the filters.

\section{MEASUREMENT RESULTS}

The following results are obtained by simulating the concept using MATLAB code.

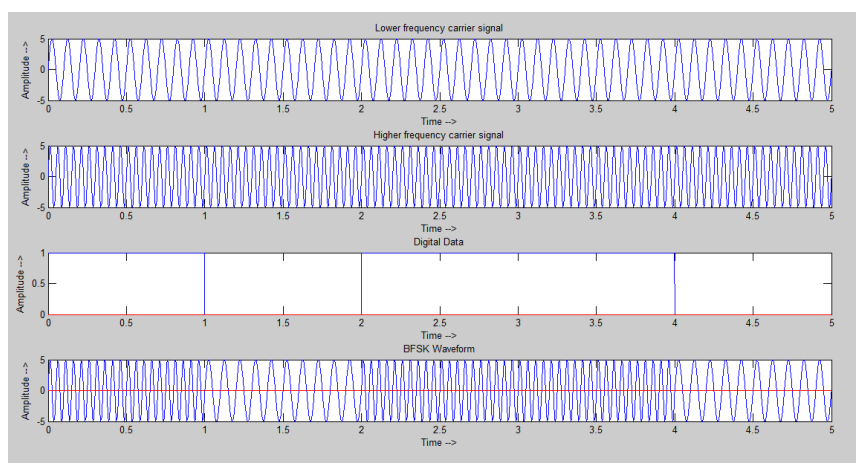

Fig -6: BFSK Modulation results on MATLAB

In figure 6, the BFSK modulation is implemented. Figure 6 (a) and Figure 6 (b) represents the lower and higher frequency carrier signal. Figure 6 (c) represents the digital data and Figure 6 (d) represents the BFSK modulated signal.

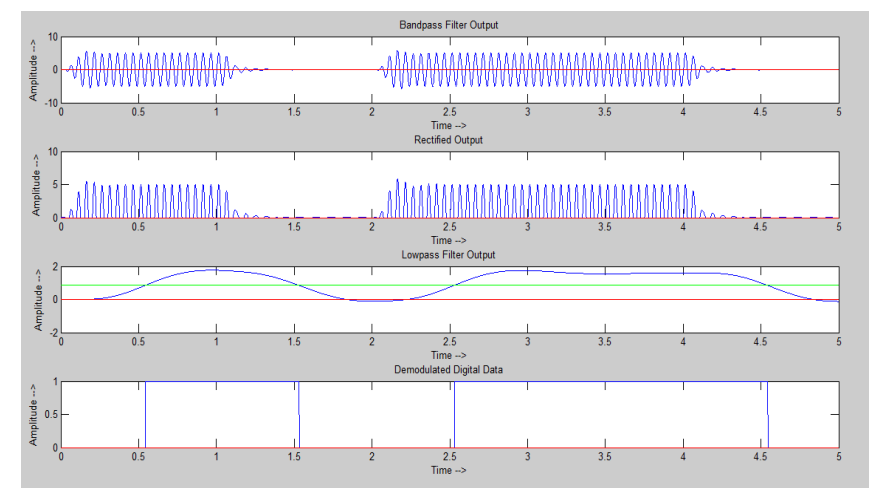

Fig -7: BFSK Demodulation results on MATLAB

In figure 7, BFSK demodulation using the proposed technique is implemented. Figure 7 (a) is the output after passing the BFSK wave from the bandpass filter. The BPF rejects the space frequency signal and the RF noise and passes the mark frequency signal effectively converting into ASK modulated waveform. Figure 7 (b) represents the rectified ASK waveform which is further passed through the Lowpass filter to smoothen out the waveform. Figure 7 (c) is the LPF output. A threshold level is set to compare the filtered waveform with this threshold level to decide 
whether a 0 or 1 was transmitted. The green line (at $1 \mathrm{~V}$ ) represents the threshold level. Figure 7 (d) is the demodulated digital data which was demodulation on the basis of the decision making comparator.

\section{CONCLUSIONS}

The BFSK demodulator can be designed using a Bandpass filter and then using a conventional ASK demodulator. This technique not only allows us to demodulate the BFSK signal but also demodulate an ASK signal using the same receiver. The idea was verified using Matrix Laboratory (MATLAB) coding. Butterworth filters were used as ripples in pass band and stop band causes error in decision making of the received bit through the comparator. Using the advanced filters, a much efficient demodulator can be designed.

\section{REFERENCES}

[1]. P.K.Dakua and P.K Nanda, Research Article Design of Telemetry System using VHDL, IJRSR Vol.5, Issue 12, pp. 2295-2297, December, 2014

[2]. Herbert Taub, Principles of Communication Systems, Second Edition, pg 272

[3]. B.P. Lathi, Modern Digital and Analog Communication Systems, $3^{\text {rd }}$ Edition, pg 340-341 